\title{
Case Report: Clinical Remission in a
} Cat With Severe Bilateral Eosinophilic Keratitis Receiving Combined Immunosuppressive Therapy (Triamcinolone Acetonide and Tacrolimus)

\author{
Amanda K. Romaneck ${ }^{1}$ and Lionel Sebbag ${ }^{1,2 *}$ \\ 'Department of Veterinary Clinical Sciences, College of Veterinary Medicine, lowa State University, Ames, IA, United States, \\ ${ }^{2}$ Koret School of Veterinary Medicine, The Hebrew University of Jerusalem, Rehovot, Israel
}

OPEN ACCESS

Edited by:

David Bruyette,

Anivive Lifesciences, United States

Reviewed by:

Shin Ae Park,

Purdue University, United States

Elena De Felice,

University of Camerino, Italy

*Correspondence: Lionel Sebbag lionel.sebbag@mail.huji.ac.il

Specialty section:

This article was submitted to Comparative and Clinical Medicine,

a section of the journal

Frontiers in Veterinary Science

Received: 06 July 2020 Accepted: 31 March 2021

Published: 30 April 2021

Citation:

Romaneck AK and Sebbag L (2021) Case Report: Clinical Remission in a Cat With Severe Bilateral Eosinophilic Keratitis Receiving Combined Immunosuppressive Therapy (Triamcinolone Acetonide and Tacrolimus). Front. Vet. Sci. 8:580396

doi: 10.3389/fvets.2021.580396
A 2-year-old domestic shorthair cat was examined for severe keratitis of 10 months duration, non-responsive to $0.1 \%$ dexamethasone g8-12h. Patient and owner compliance were poor given ocular discomfort and hiding behavior. On presentation, both eyes (OU) had severe ulcerative keratitis that masked examination of deeper structures and resulted in absent menace response OU. Corneal cytology was consistent with eosinophilic keratitis (EK) OU. Initial therapy included subcutaneous triamcinolone acetonide injection $(0.2 \mathrm{mg} / \mathrm{kg}), 0.3 \%$ ciprofloxacin OU q12h, and two compounded drugs started 5 days later upon receipt: $0.5 \%$ tacrolimus OU q6h, 0.5\% cidofovir OU q12h. Visual behavior and ocular comfort were reportedly much improved within $24 \mathrm{~h}$. At the first recheck (Day 11), patient and owner compliance were reported to be excellent, menace response was positive $\mathrm{OU}$, and keratitis was dramatically reduced $\mathrm{OU}$ with absent fluorescein uptake. Subcutaneous triamcinolone acetonide was repeated (0.2 $\mathrm{mg} / \mathrm{kg}$ ), ciprofloxacin was discontinued, cidofovir was continued $\mathrm{q} 12 \mathrm{~h}$, and tacrolimus was slowly tapered (q8h $\times 3$ weeks then q12h). Keratitis was nearly $100 \%$ resolved at the second recheck (Day 38); cidofovir was discontinued and tacrolimus was slowly tapered ( $\mathrm{q} 12 \mathrm{~h} \times 1$ week, $\mathrm{q} 24 \mathrm{~h} \times 4$ weeks, $\mathrm{q} 48 \mathrm{~h} \times 4$ weeks) then discontinued. A third recheck (Day 101) confirmed clinical remission OU, and disease did not reoccur within a 1 year follow up period (photographic documentation by owner). In sum, adjunct use of triamcinolone acetonide greatly improved therapeutic compliance and helped control severe EK in a rapid and effective manner.

Keywords: eosinophilic keratitis, eosinophilic keratoconjunctivitis, triamcinolone acetonide, corticosteroids, immunomodulation, antiviral drugs, compliance

\section{BACKGROUND}

Eosinophilic keratitis (EK) is a chronic and progressive keratopathy described in cats and other species, characterized by proliferative white to pink granular corneal plaques and corneal neovascularization (1-5). The exact pathogenesis of EK is unknown, although an association with feline herpesvirus type 1 (FHV-1) has been proposed in cats (6), and the cellular profile 
is typically consistent with either a type I (IgE-mediated) or type IV (T-lymphocyte-mediated) hypersensitivity reaction (7).

Diagnosis of EK can be confirmed with corneal cytologyrevealing a predominantly eosinophilic inflammation-while treatment of EK is aimed at controlling the immune-mediated disease with the use of topical and/or systemic immunemodulatory drugs. Several challenges complicate the clinical management of EK in cats, including the balance between local immunosuppression and FHV-1 reactivation (8), risk of topical corticosteroids in the presence of corneal ulceration (reported in $66.7 \%$ of EK cats) (3), poor therapeutic compliance long-term (2), and relatively high rate of recurrences (up to 66\%) (1).

The purpose of the present case report is to describe the successful management of severe bilateral EK in a cat, detailing a unique protocol that enhanced therapeutic compliance, improved clinical signs in a rapid manner, and provided long-term resolution without maintenance immunomodulation (clinical remission at 1 year follow up).

\section{CASE PRESENTATION}

Written informed consent was obtained from the owner for the publication of this case report. A 2-year-old male castrated domestic shorthair $(7 \mathrm{~kg})$ presented for evaluation of keratitis of approximately 10 months duration. The cat was otherwise healthy with no known systemic diseases, and he was up-todate on vaccines and flea and tick prevention. An opacity was first noted by the owner in the lateral aspect of the cornea in the left eye (OS), followed weeks later by similar changes in the right eye (OD). The lesions slowly extended over the entire corneal surface in both eyes (OU) over several months, resulting in visual impairment, blepharospasm, ocular discharge, and redness. According to the owner, the visual impairment affected the cat's general demeanor (i.e., hiding behavior, easily startled) and started disrupting the human-animal bond in the household. Two months prior to presentation, the cat was prescribed topical $0.1 \%$ dexamethasone (Maxidex, Novartis, Basel, Switzerland) by the referring veterinarian with a recommended administration frequency of three times daily OU. However, therapy was only given twice daily by the owner due to the patient's ocular discomfort and poor compliance, and no improvement was noted at the completion of the 3-week course.

On presentation to a board-certified veterinary ophthalmologist (LS), physical examination and vital parameters were within normal limits. The cat appeared hesitant in the examination room (difficulty navigating, unable to track cotton balls) and the menace response was absent OU. Dazzle and palpebral reflexes were intact although pupillary light reflexes were not able to be assessed due to the severity of the corneal opacification OU. Mild blepharospasm and seromucoid to mucopurulent discharge were present $\mathrm{OU}$. The conjunctiva had diffuse moderate hyperemia and mild chemosis OU. Dense fibrovascular ingrowth affected $100 \%$ of the anterior cornea $\mathrm{OU}$, in addition to white raised plaques present on the corneal surface OU (masked by the third eyelid OD) (Figures 1A,B). Ocular diagnostic testing showed that intraocular pressures
(TonoVet, Jorgensen Laboratories) were normal (23 mmHg OD, $24 \mathrm{mmHg}$ OS), Schirmer tear test-1 (Merck Animal Health) results were normal $(16 \mathrm{~mm} / \mathrm{min} \mathrm{OD}, 19 \mathrm{~mm} / \mathrm{min}$ OS) $(9,10)$, and multifocal punctate areas of fluorescein uptake were noted OU. Cytological evaluation of the white corneal plaques revealed a mixed, predominately eosinophilic inflammation with few mast cells and lymphocytes (Figure 2). Clinical diagnoses were severe EK with punctate corneal ulcerations and moderate conjunctivitis OU. Treatment was initiated with triamcinolone acetonide (Vetalog, Boehringer Ingelheim Vetmedica, St. Joseph, $\mathrm{MO}$ ) administered subcutaneously at $0.2 \mathrm{mg} / \mathrm{kg}$, as well as $0.3 \%$ ciprofloxacin ophthalmic solution (Ciloxan, Alcon Laboratories, Fort Worth, TX; 1 drop OU q12h) and two topical compounded medications started 5 days later upon receipt: $0.5 \%$ tacrolimus aqueous solution (Stokes pharmacy, Mount Laurel, NJ; 1 drop OU q6h) and $0.5 \%$ cidofovir aqueous solution (Stokes Pharmacy, Mount Laurel, NJ; 1 drop OU q12h).

On Day 1 (i.e., $24 \mathrm{~h}$ following triamcinolone injection), telephonic update with the owner revealed that the cat's visual behavior and ocular comfort were greatly improved, as well as the tolerance to handling for topical drug administration. On Day 11, recheck examination showed marked improvement in vision (navigation in exam room, intact menace response $\mathrm{OU}$ ) and ocular comfort level (no blepharospasm), with notable reduction in ocular discharge and conjunctivitis severity $\mathrm{OU}$ (mild residual hyperemia). Keratitis was greatly improved with resolution of corneal plaques and epithelial defects OU (no fluorescein uptake), as well as marked reduction in the fibrovascular ingrowth (mild residual corneal vascularization) allowing for clear visualization of the inside of the eye $\mathrm{OU}$ (Figures 1C,D). A subcutaneous injection of triamcinolone acetonide $(0.2 \mathrm{mg} / \mathrm{kg})$ was repeated, $0.3 \%$ ciprofloxacin was discontinued, $0.5 \%$ cidofovir was continued $\mathrm{q} 12 \mathrm{~h}$ for 2 weeks, and $0.5 \%$ tacrolimus was slowly tapered (q8h for 3 weeks then q12h until recheck).

Recheck evaluation on Day 38 revealed resolution of conjunctivitis and ocular discharge, and near resolution of the keratitis OU (Figures 1E,F). Only thin superficial vessels were present in the cornea $\mathrm{OU}$, most of which were hypoperfused ("ghost" vessels). Intraocular pressures $(20 \mathrm{mmHg}$ OD, $25 \mathrm{mmHg}$ OS) and Schirmer tear test-1 results $(20 \mathrm{~mm} / \mathrm{min}$ OD, 25 $\mathrm{mm} / \mathrm{min}$ OS) were normal. At home treatment involved $0.5 \%$ tacrolimus at q12h for 1 week, then q24h for 4 weeks, then $\mathrm{q} 48 \mathrm{~h}$ with instructions to discontinue administration 1 week before the following recheck.

The cat was re-examined on Day 101, off any medication for 5 days. The recheck visit confirmed resolution of ocular signs OU with only few hypoperfused superficial corneal vessels noticed on retro-illumination (Figures $\mathbf{1 G}, \mathbf{H}$ ). Throughout all visits, no systemic adverse effects (e.g., flare up of herpetic disease such as sneezing or nasal discharge) or ocular irritation from compounded tacrolimus were reported by the owner. Further, phone and email communications with the owner confirmed long-term clinical remission (i.e., no recurrence of ocular surface disease) despite discontinuation of all therapies. The latest follow-up at time of manuscript writing was Day 374 (Figures 3, 4). 

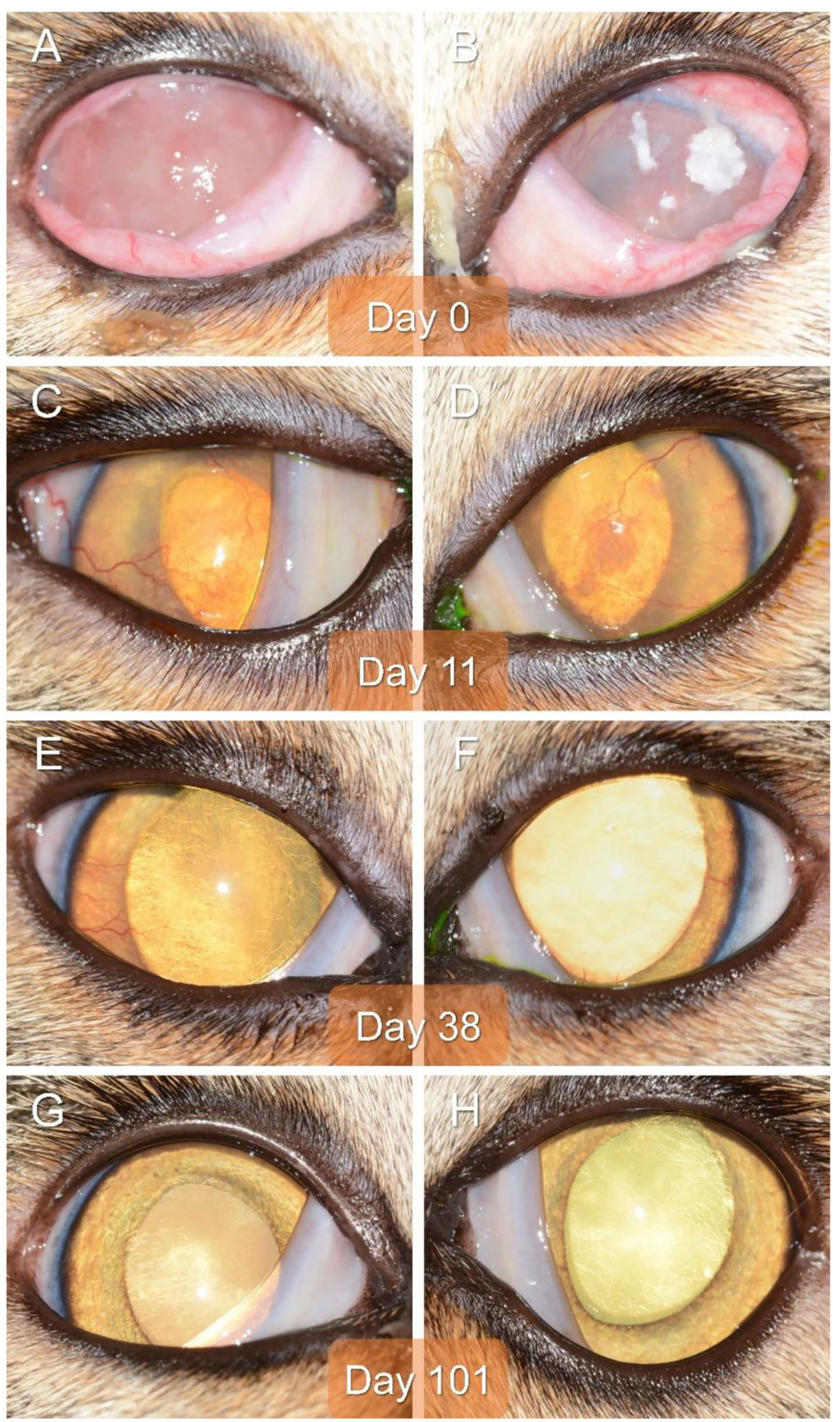

FIGURE 1 | Clinical images of the right eye (A,C,E,G) and left eye (B,D,F,H) of a 2-year-old domestic shorthair cat diagnosed with severe bilateral eosinophilic keratitis, managed with combined immunosuppressive therapy (subcutaneous triamcinolone acetonide and topical 0.5\% tacrolimus), and examined on Day 0 (A,B), Day 11 (C,D), Day 38 (E,F), and Day 101 (G,H). 


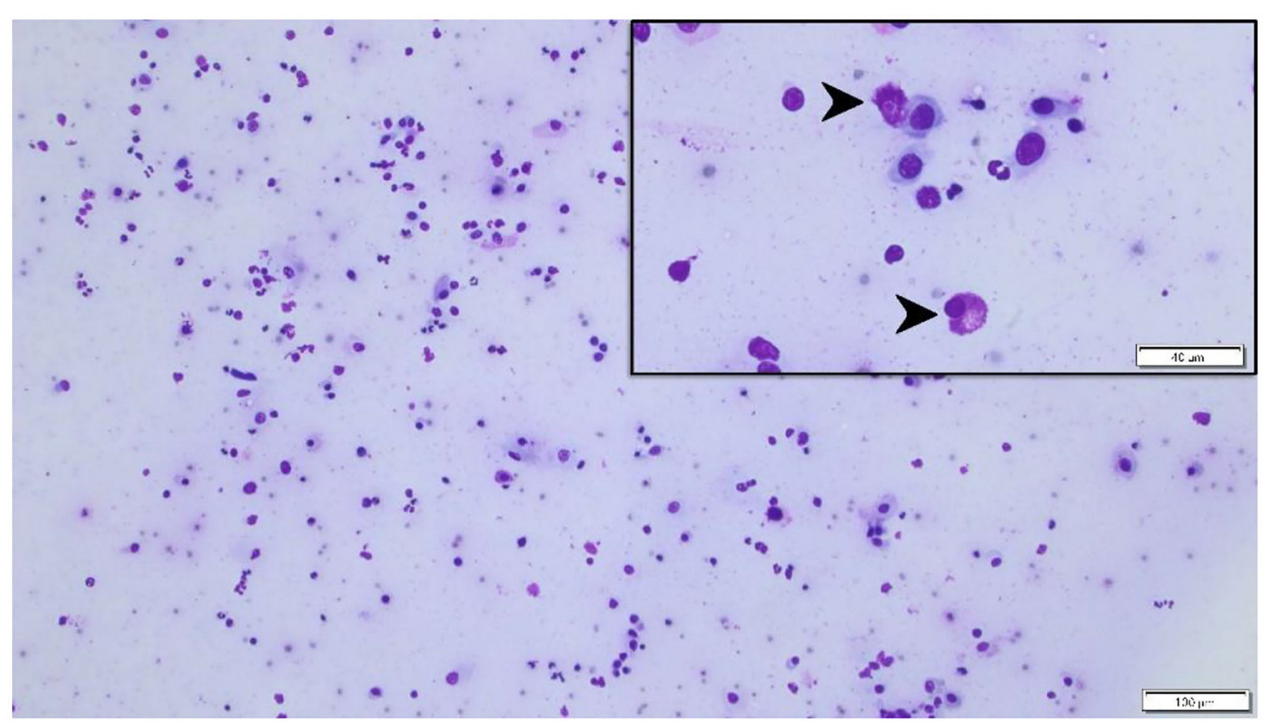

FIGURE 2 | Cytological specimen of the left corneal surface (Wright Giemsa stain) highlighting a mixed, predominantly eosinophilic inflammation (black arrowheads) with few mast cells and lymphocytes. Bar $=40 \mu \mathrm{m}$ (top right insert) or $100 \mu \mathrm{m}$ (background image).
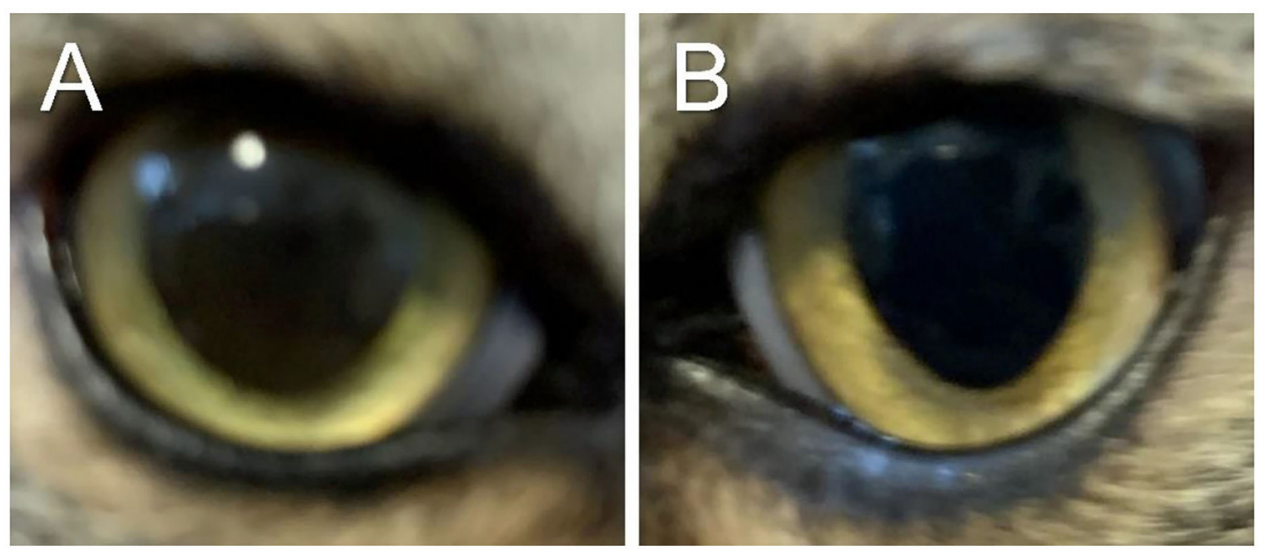

FIGURE 3 | Clinical images of the right eye (A) and left eye (B) of a cat with historical eosinophilic keratitis in clinical remission. Images were taken by the owner using a smartphone on Day 374 following initial diagnosis, that is, 278 days after discontinuation of all therapies.

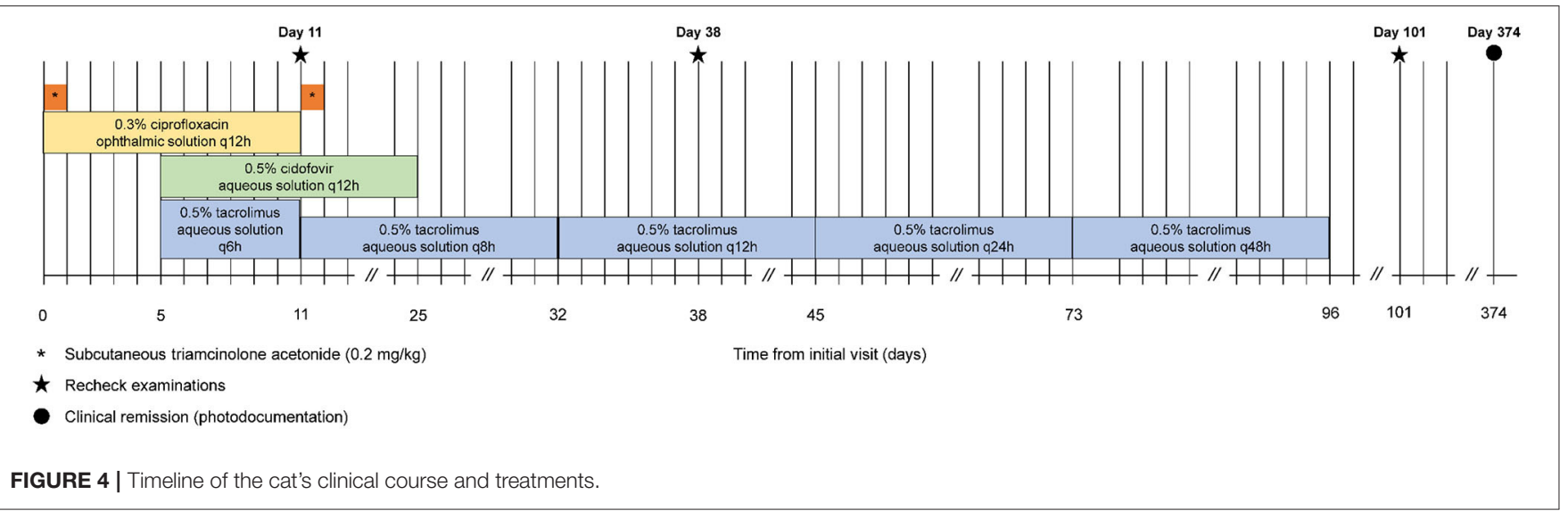




\section{DISCUSSION}

The present case report describes the successful management of severe EK in both eyes of a cat, highlighting two important features: (i) Use of triamcinolone acetonide as the primary method of immunomodulation (rather than conventional topical immunotherapy) to improve therapeutic compliance; and (ii) Clinical remission with no recurrence of keratitis despite discontinuation of immunomodulation after merely 3.2 months of therapy.

Triamcinolone acetonide is an intermediate acting glucocorticoid with negligible mineralocorticoid effects, considered approximately 7 times as potent as methylprednisone (11). Parenteral administration of triamcinolone provides corticosteroid effects for 7-15 days, and a second dose can be administered following this time if signs persist (12). Here, triamcinolone acetonide was injected subcutaneously at two visits separated by 11 days, providing a rapid and marked improvement in clinical signs and ocular comfort. Our findings are consistent with a study of EK in horses, where systemic corticosteroid use was associated with a significantly shorter time for resolution of clinical signs (4). Our work is also complementary to the recent study by Lucyshyn et al., in which subcutaneous administration of triamcinolone was deemed safe and as efficacious as conventional topical immunomodulation in cats with eosinophilic keratoconjunctivitis (13). However, two main differences exist between the two reports: (i) First, clinical remission (noted in our patient) was not described in any cat reported by Lucyshyn et al., a finding that could be partly explained by the higher intensity of topical therapy in this case report (i.e., high concentration and frequency of tacrolimus); (ii) Second, triamcinolone was administered at time of diagnosis in our feline patient, whereas triamcinolone was preceded by a course of antiviral therapy with famciclovir or $0.5 \%$ cidofovir (median 17 days, range 0-63 days) in Lucyshyn's study. The tentative goal of initiating antiviral therapy prior to corticotherapy was to reduce the likelihood of herpetic flare-up and corneal ulceration, however the authors acknowledged that simultaneous administration of triamcinolone and antiviral drug may be preferred (as described herein) to reduce time to disease resolution, cost, and number of recheck visits.

There are several notable advantages to the unique protocol described in our study. First, triamcinolone acetonide provided rapid and pronounced improvement in corneal inflammation, allowing for corneal epithelial defects to heal without the risks associated with topical immunomodulation (i.e., potentiation of infection, delayed re-epithelialization). Second, medication compliance from the patient (and consequently owner) was greatly enhanced by reducing ocular discomfort before initiation of topical medications at high frequency; indeed, administration of multiple eyedrops 2-3 times daily (or more) can be particularly challenging in a cat that is painful and hiding from the owner. Compliance is particularly relevant for EK as most cases of recurrences were reportedly associated with poor mediation adherence (2). In the present case, the owner struggled medicating the cat prior to the first visit, then reported excellent compliance as early as Day 1 given the substantial improvement in the cat's ocular comfort and general demeanor. Third, corticosteroid levels on the ocular surface are likely optimized with triamcinolone acetonide as compared to topical corticosteroid use; the former provides a controlled-release formulation that achieves sustained concentrations over an extended period (zero-order kinetics), while the latter provides high concentrations at delivery but rapid decline due to efficient drug removal via the nasolacrimal drainage apparatus (firstorder kinetics) $(14,15)$. Systemic corticosteroid administration achieved quantifiable tear film concentrations in dogs (16), and the same is likely true in cats. Further research is needed to characterize triamcinolone pharmacokinetics in cats, assessing healthy subjects but also cats with conjunctivitis given the likelihood for higher tear film concentrations in eyes with compromised blood-tear barrier $(17,18)$.

Similar to other reports of cats receiving triamcinolone acetonide for EK or other conditions $(13,19)$, our patient did not experience notable adverse effects from systemic corticotherapy. However, this observation should be verified in future prospective studies that include systemic workup and diagnostic testing. Potential adverse effects of systemic corticotherapy in cats include (but not limited to) recrudescence of herpetic disease (20), plasma volume expansion (21) that could promote congestive heart failure in predisposed cats (22), and rarely, iatrogenic hyperadrenocorticism (23).

Maintenance immunotherapy was achieved with topical 0.5\% tacrolimus aqueous solution, following an initial frequency of four times daily that was slowly tapered off over 3 months. Of note, the prescribed concentration $(0.5 \%)$ was much higher than the typical dose of tacrolimus reported in previous ophthalmic studies $(0.02 \%)$ (24), with the aim to provide an aggressive immunosuppressive therapy from the onset of medical management (discussed below). Further, tacrolimus was preferred over previously described $1.5 \%$ cyclosporine (2) for two reasons: (i) Tacrolimus is more potent than cyclosporine for reducing corneal neovascularization in patients with severe keratitis, as reported in dogs with keratoconjunctivitis sicca (24); and (ii) aqueous-based compounded tacrolimus is subjectively better tolerated than oil-based compounded cyclosporine in cats (authors' personal experience), thus reducing the risk of stopping therapy due to local adverse effects such as marginal blepharitis (2).

Monotherapy is generally effective in managing EK in cats, as described for topical $1.5 \%$ cyclosporine (2) or $0.5 \%$ megestrol acetate (5), although this approach is limited by incomplete success rate (11.4 and $12 \%$ non-responsive, respectively), relatively slow clinical improvement ( $>2-3$ weeks), disease recurrence (22.6 and 33\%, respectively), and the need to maintain cats on immunomodulation long-term to lifelong $(2,5)$. In contrast, the use of combined immunosuppressive drugs in the present case (systemic triamcinolone and topical tacrolimus) achieved a rapid improvement of EK ( $<11$ days) that resulted in clinical remission following discontinuation of all medications by 3.2 months. There is mounting evidence that early and aggressive immunomodulation improves longterm clinical outcomes and disease prognosis in humans with diverse immune-mediated diseases (e.g., rheumatoid arthritis, 
Crohn's disease). For instance, human patients with Crohn's disease were significantly more likely to achieve clinical remission when receiving combination immunosuppressive therapy (60\%) rather than conventional therapy with incremental use of immunomodulation drugs (35.9\%) (25). The same may be true in cats with EK given the immune-mediated pathogenesis of this ocular condition $(2,5)$, although the findings of the present case report should be verified in future prospective controlled studies assessing a larger population of cats.

The main limitation of the study was the lack of diagnostic testing for FHV-1. FHV-1 is detected in over $76 \%$ of corneal scrapings from cats with EK (6), and its presence can complicate medical management due to potential herpetic flare up with immunosuppressive drug. The patient had historical upper respiratory infections during kittenhood, thus $0.5 \%$ cidofovir was initiated empirically to reduce the risk for potential FHV-1 reactivation despite the lack of confirmatory testing. Cidofovir was well-tolerated and discontinued 2 weeks after re-epithelialization of corneal ulcers.

\section{CONCLUDING REMARKS}

The case report highlights the use of combination immunosuppressive therapy to achieve rapid resolution of clinical signs and greater chance for full recovery (clinical remission). Subcutaneous administration of triamcinolone acetonide improved ocular comfort and therapeutic

\section{REFERENCES}

1. Morgan VR. Feline eosinophilic keratitis: a retrospective study of 54 cases:(1989-1994). Vet Comp Ophthalmol. (1996) 6:131-4.

2. Spiess AK, Sapienza JS, Mayordomo A. Treatment of proliferative feline eosinophilic keratitis with topical 1.5\% cyclosporine: 35 cases. Vet Ophthalmol. (2009) 12:132-7. doi: 10.1111/j.1463-5224.2008.00679.x

3. Dean E, Meunier V. Feline eosinophilic keratoconjunctivitis: a retrospective study of 45 cases (56 eyes). J Feline Med Surg. (2013) 15:661-6. doi: 10.1177/1098612X12472181

4. Lassaline-Utter M, Miller C, Wotman KL. Eosinophilic keratitis in 46 eyes of 27 horses in the Mid-Atlantic United States (2008-2012). Vet Ophthalmol. (2014). 17:311-20. doi: 10.1111/vop.12076

5. Stiles J, Coster M. Use of an ophthalmic formulation of megestrol acetate for the treatment of eosinophilic keratitis in cats. Vet Ophthalmol. (2016) 19(Suppl 1):86-90. doi: 10.1111/vop.12371

6. Nasisse MP, Glover TL, Moore CP, Weigler BJ. Detection of feline herpesvirus 1 DNA in corneas of cats with eosinophilic keratitis or corneal sequestration. Am J Vet Res. (1998) 59:856-8.

7. Prasse KW. Cytology and histopathology of feline eosinophlic keratitis. Vet Comp Ophthalmol. (1996) 6:74-81.

8. Nasisse MP. Anti-inflammatory therapy in herpesvirus keratoconjunctivitis. Prog Vet Comp Ophthalmol. (1991) 1:63-5.

9. Sebbag L, Kass PH, Maggs DJ. Reference values, intertest correlations, and test-retest repeatability of selected tear film tests in healthy cats. J Am Vet Med Assoc. (2015) 246:426-35. doi: 10.2460/javma.246.4.426

10. Sebbag L, Uhl LK, Schneider B, Hayes B, Olds J, Mochel JP. Investigation of Schirmer tear test-1 for measurement of tear production in cats in various environmental settings and with different test durations. J Am Vet Med Assoc. (2020) 256:681-6. doi: 10.2460/javma.256.6.681 compliance-a key component for long-term successand resulted in excellent disease control in a rapid and effective manner.

\section{DATA AVAILABILITY STATEMENT}

The raw data supporting the conclusions of this article will be made available by the authors, without undue reservation.

\section{ETHICS STATEMENT}

Ethical review and approval was not required for the animal study because the study describes the clinical management of a patient for a routine ocular condition. Written informed consent was obtained from the owner for the participation of their animal in this study.

\section{AUTHOR CONTRIBUTIONS}

LS examined the patient and conceived the medical management used in the study. AR and LS wrote the manuscript. All authors contributed to the article and approved the submitted version.

\section{ACKNOWLEDGMENTS}

The authors are thankful to the UMN ophthalmology service (Dr. Marina Leis, Stacy Bokelheide, and Melissa Boyd) for their assistance with the patient's care.
11. Ganz EC, Griffin CE, Keys DA, Flatgard TA. Evaluation of methylprednisolone and triamcinolone for the induction and maintenance treatment of pruritus in allergic cats: a doubleblinded, randomized, prospective study. Vet Dermatol. (2012) 23:387-e72. doi: 10.1111/j.1365-3164.2012.01058.x

12. Ingelheim B. Vetalog Parenteral (Package Insert). St Joseph, MO: Boehringer Ingelheim Vetmedica Inc. (2014).

13. Lucyshyn DR, Good KL, Knickelbein KE, Chang MW, Strøm AR, Hollingsworth SR, et al. Subcutaneous administration of triamcinolone as part of the management of feline eosinophilic keratoconjunctivitis. $J$ Feline Med Surg. (2020) 2020:1098612X20968660. doi: 10.1177/1098612X209 68660

14. Sebbag L, Allbaugh RA, Wehrman RF, Uhl LK, Ben-Shlomo G, Chen $\mathrm{T}$, et al. Fluorophotometric assessment of tear volume and turnover rate in healthy dogs and cats. J Ocul Pharmacol Ther. (2019) 35:497502. doi: 10.1089/jop.2019.0038

15. Sebbag L, Kirner NS, Allbaugh RA, Reis A, Mochel JP. Kinetics of fluorescein in tear film after eye drop instillation in beagle dogs: does size really matter? Front Vet Sci. (2019) 6:457. doi: 10.3389/fvets.2019.00457

16. Sebbag L, Yan Y, Smith JS, Allbaugh RA, Wulf LW, Mochel JP. Tear fluid pharmacokinetics following oral prednisone administration in dogs with and without conjunctivitis. J Ocul Pharmacol Ther. (2019) 35:3419. doi: 10.1089/jop.2019.0020

17. Sebbag L, Allbaugh RA, Weaver A, Seo YJ, Mochel JP. Histamineinduced conjunctivitis and breakdown of blood-tear barrier in dogs: a model for ocular pharmacology and therapeutics. Front Pharmacol. (2019) 10:752. doi: 10.3389/fphar.2019.00752

18. Sebbag L, Mochel JP. An eye on the dog as the scientist's best friend for translational research in ophthalmology: focus on the ocular surface. Med Res Rev. (2020) 40:2566-04. doi: 10.1002/med.21716 
19. Momota Y, Yasuda J, Arai N, Yamamoto M, Yoshimura H, Ikezawa M, et al. Contribution of oral triamcinolone to treating proliferative and necrotising otitis externa in a 14-year-old Persian cat. JFMS Open Rep. (2017) 3:2055116917691175. doi: 10.1177/2055116917691175

20. Gaskell RM, Povey RC. Re-excretion of feline viral rhinotracheitis virus following corticosteroid treatment. Vet Rec. (1973) 93:2045. doi: 10.1136/vr.93.7.204

21. Block CL, Oyama MA. Echocardiographic and biomarker evidence of plasma volume expansion after short-term steroids administered orally in cats. $J$ Vet Intern Med. (2020) 34:29-34. doi: 10.1111/jvim.15678

22. Smith SA, Tobias AH, Fine DM, Jacob KA, Ployngam T. Corticosteroidassociated congestive heart failure in 12 cats. Intern J Appl Res Vet Med. (2004) 2:159-70.

23. Lien $\mathrm{YH}, \mathrm{Huang} \mathrm{HP}$, Chang $\mathrm{PH}$. Iatrogenic hyperadrenocorticism in 12 cats. $J$ Am Anim Hosp Assoc. (2006) 42:414-23. doi: 10.5326/0420414

24. Radziejewski K, Balicki I. Comparative clinical evaluation of tacrolimus and cyclosporine eye drops for the treatment of canine keratoconjunctivitis sicca. Acta Vet Hung. (2016) 64:313-29. doi: 10.1556/004 2016.030

25. D'Haens G, Baert F, van Assche G, Caenepeel P, Vergauwe P, Tuynman H, et al. Early combined immunosuppression or conventional management in patients with newly diagnosed Crohn's disease: an open randomised trial. Lancet. (2008) 371:660-7. doi: 10.1016/S0140-6736(08)60304-9

Conflict of Interest: The authors declare that the research was conducted in the absence of any commercial or financial relationships that could be construed as a potential conflict of interest.

Copyright (C) 2021 Romaneck and Sebbag. This is an open-access article distributed under the terms of the Creative Commons Attribution License (CC BY). The use, distribution or reproduction in other forums is permitted, provided the original author(s) and the copyright owner(s) are credited and that the original publication in this journal is cited, in accordance with accepted academic practice. No use, distribution or reproduction is permitted which does not comply with these terms. 